\title{
Ulnar impaction syndrome: A case series and imaging approach
}

\author{
Dr Cuan Liebenberg MBChB \\ Department of Radiology, University of Pretoria \\ Correspondence: \\ Dr Farhana E Suleman \\ Department of Radiology \\ University of Pretoria \\ Email: fesuleman@gmail.com \\ Tel: 0123252406 \\ Department of Radiology \\ Private Bag X169 \\ 0001 Pretoria
}

Dr Mark D Velleman MBChB, FCRad(D)(SA), MMedRad(D)

Dr Farhana E Suleman MBChB, FCRad(D)(SA), MMedRad(D)

\begin{abstract}
Ulnar-sided wrist pain can be attributed to many pathological processes. This can include traumatic, inflammatory or degenerative conditions. Ulnar impaction syndrome is a group of syndromes that are degenerative conditions of the wrist caused by an abnormal joint configuration or due to abnormal use. This leads to an increase in axial loading across the ulnar side of the wrist with resultant joint degeneration. The structures in the wrist concerned in this syndrome are the triangular fibrocartilage complex, the distal radio-ulnar joint and the lunate triquetral bones at their ulnar articulations. Even though a number of modalities exist to image the wrist, the options for accurately assessing ulnar impaction syndrome are limited and may be challenging. Accurate assessment of the triangular fibro-cartilage complex is essential, as it lies central in the classification of the disease.
\end{abstract}

Key words: ulnar impaction syndrome, triangular fibrocartilage complex, wrist pain

\section{Introduction}

Ulnar-sided wrist pain can be attributed to many pathological processes whether traumatic, inflammatory or degenerative in nature. The patient with an acute traumatic incident can usually easily be identified from the history but others may present with non-specific complaints of intermittent wrist pain or swelling, sometimes aggravated by certain actions, or stiffness. Often the only positive finding may be a degree of tenderness or joint swelling. This clinical scenario may create a diagnostic and therapeutic dilemma.

We report a series of four patients with histories ranging from intermittent ulnar-sided wrist pain and swelling to persistent pain on the ulnar side of the wrist, and discuss the impaction syndromes with the emphasis on imaging of the condition.

\section{Case reports}

Case 1

A 50-year-old female presented with persistent ulnar-sided wrist pain on the right and a previous history of dorsal ganglion excision. MRI revealed neutral ulnar variance with a slightly dorsally tilted ulna. The proton density fat suppressed (PDFS) coronal image demonstrated inflammatory changes and oedema in the distal radio-ulnar joint (Figure 1a) with bone oedema in the proximal ulnar pole of the lunate (Figure 1b). There was some sclerosis of the lunate noted on the T1-weighted study. There was fraying and irregularity of the ulnar attachment of the triangular fibrocartilage (TFC) with partial tearing also demonstrated on the PDFS coronal study (Figure 1c). The features were in keeping with ulnar impaction syndrome. 

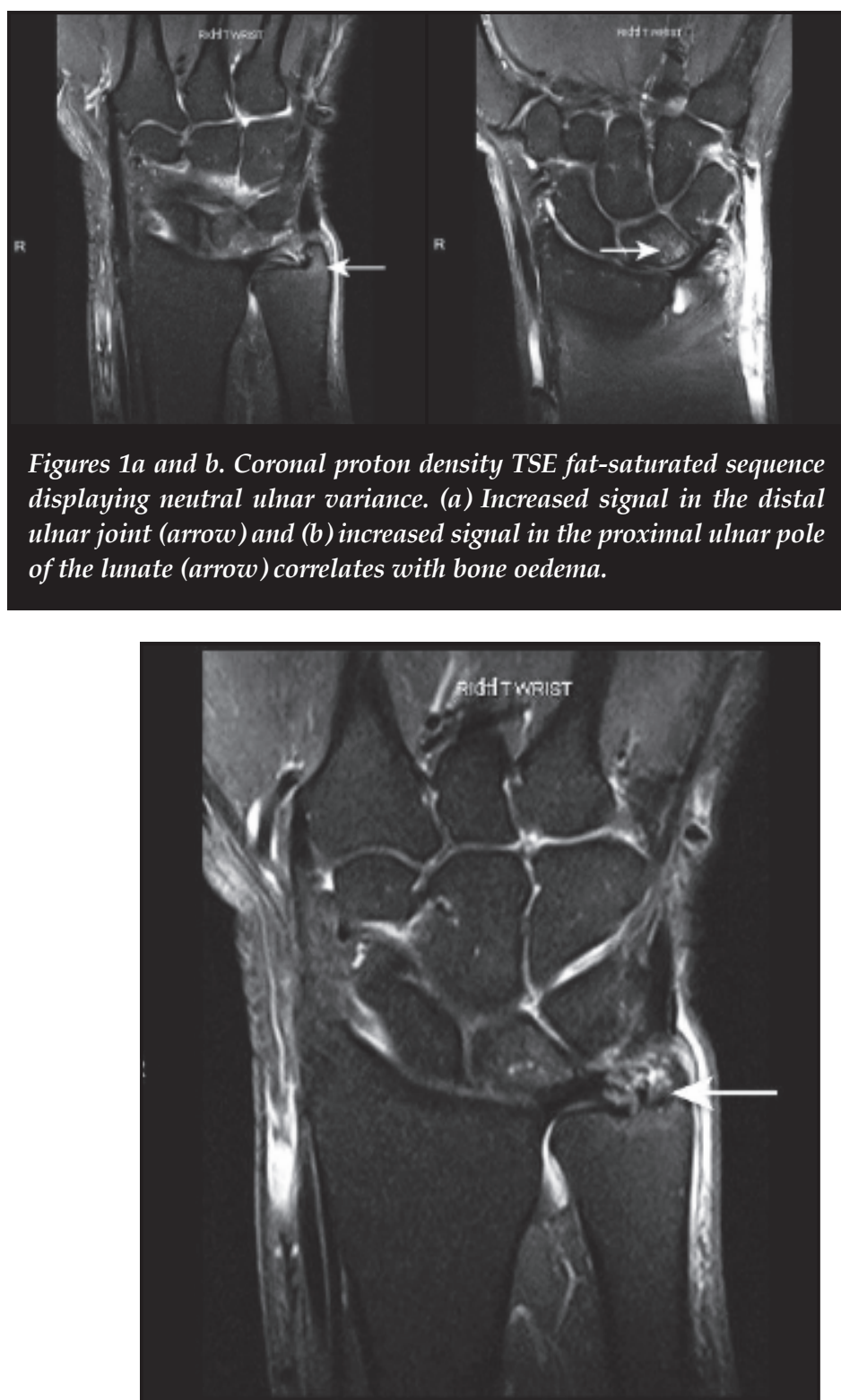

Figure 1c. Coronal proton density fat saturated sequence also displays fraying and irregularity of the ulnar attachment of the TFC with partial tear noted (arrow)

\section{Case 2}

A 42-year-old female presented with pain in the right wrist and intermittent ulnar-sided swelling. Plain film radiographs confirmed a neutral ulnar variance. The MRI demonstrated subtle sclerosis of the ulna and proximal pole of the lunate (Figure 2a), especially at the volar aspect, with minimal punctate high signal on the PDFS coronal study in keeping with early cystic changes (Figure 2b). The MRI findings were in keeping with subtle signs of chronic ulnar lunate impaction.

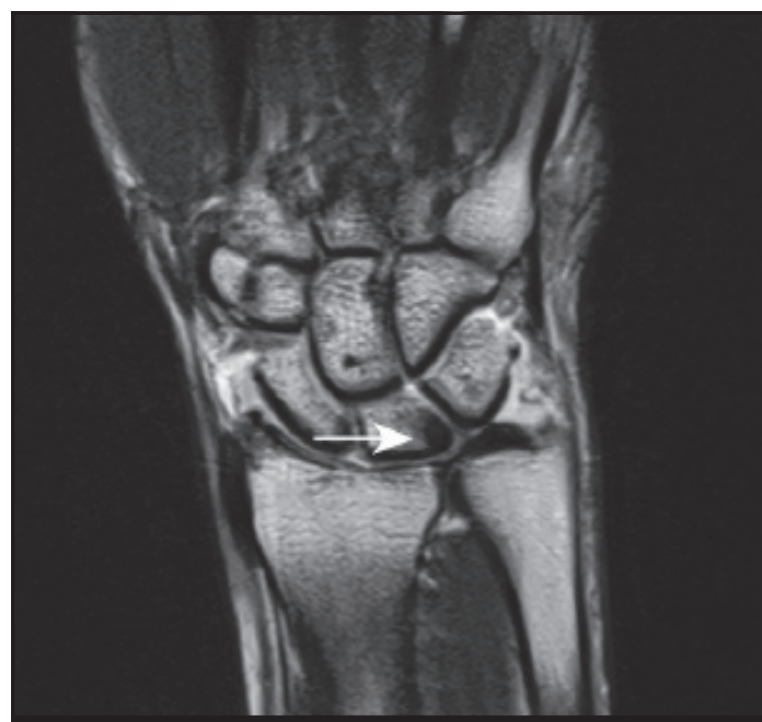

Figure 2a. Coronal proton density TSE sequence demonstrating low signal in the proximal ulnar pole of the lunate in keeping with sclerosis (arrow)

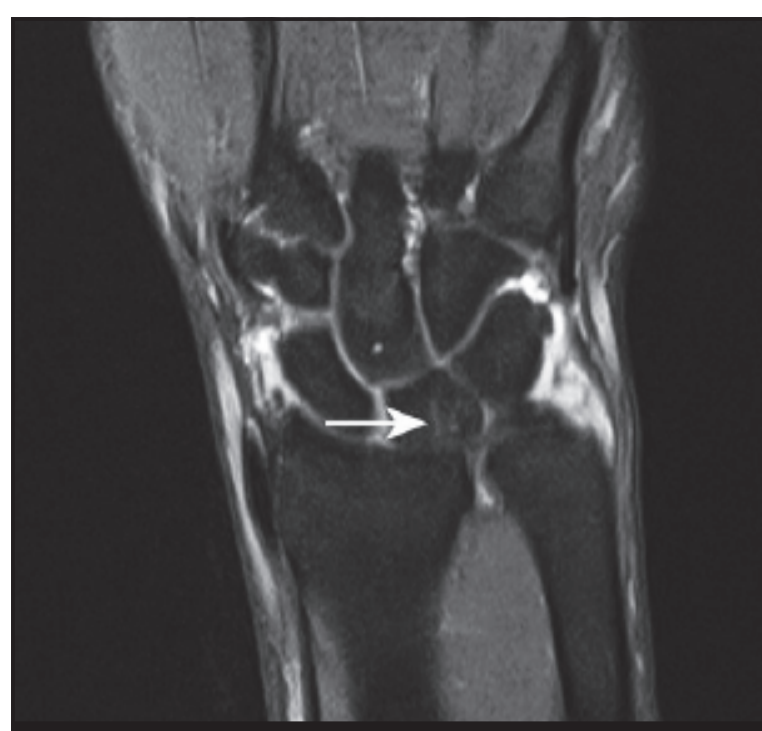

Figure 2b. Coronal proton density fat suppressed TSE sequence demonstrating punctate high signal in the proximal lunate in keeping with cystic change (arrow)

\section{Case 3}

A 34-year-old female with a history of sports training presented with ulnar-sided right wrist pain. Neutral ulnar variance was noted on plain film and MRI. Radial perforation of the triangular fibrocartilage complex (TFCC) with leakage of contrast into the distal radio-ulnar space was noted on T1-weighted coronal fat suppressed image (Figure $3 a)$. There were also impaction changes and sclerosis involving the ulna and proximal pole of the lunate which could be attributed to her sporting activity (Figure $3 b$ ). 


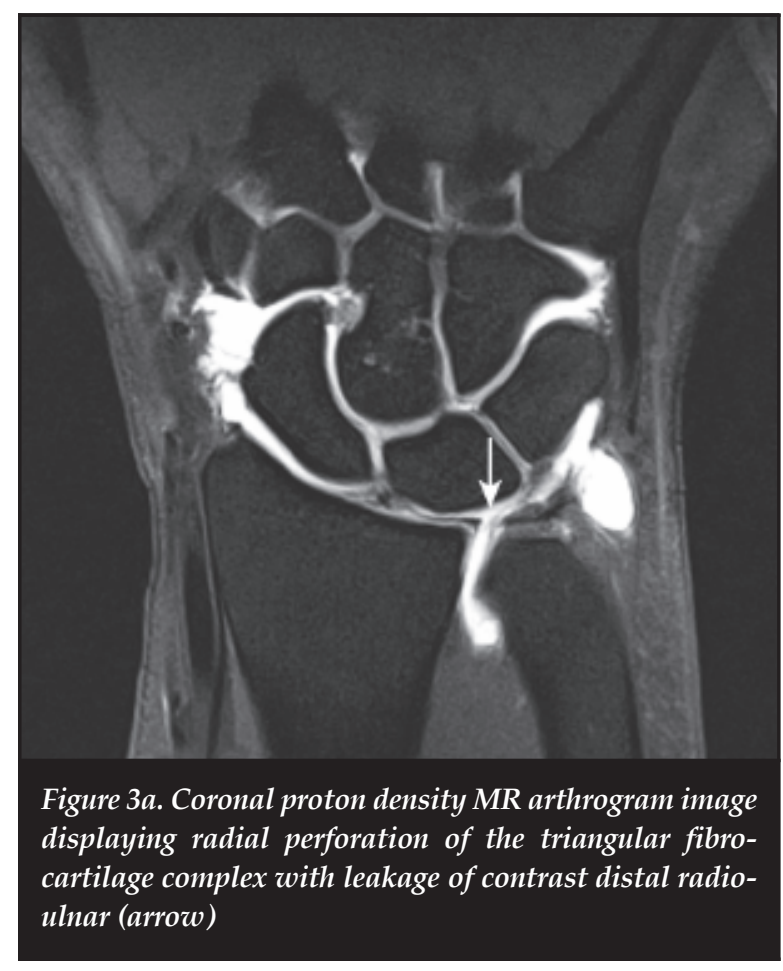

A diagnosis of ulnar impaction syndrome was made on imaging.

\section{Case 4}

A 47-year-old male presented with history of left wrist pain. The plain film radiographs demonstrated a mildly

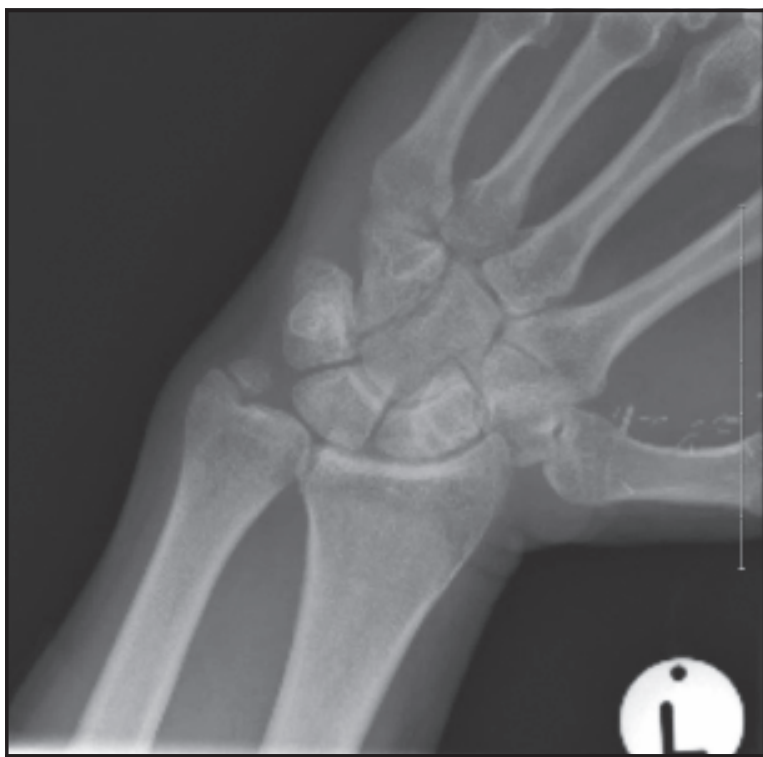

Figure 4a. Frontal radiograph of the left wrist indicating slightly positive ulna variance as well as an old ulnar styloid process fracture with nonunion

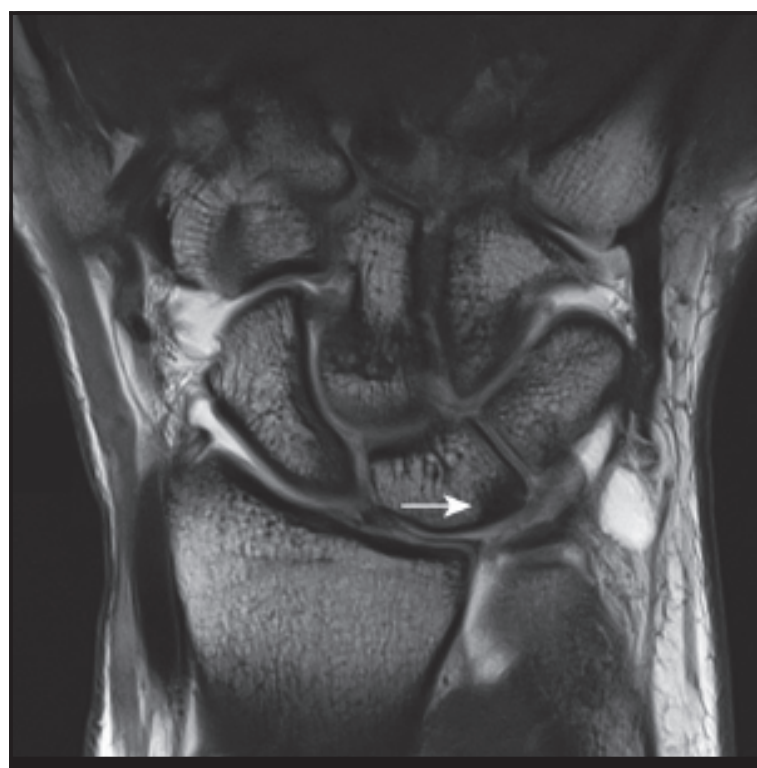

Figure 3b. Coronal proton density sequences displaying decreased signal in the proximal pole of the lunate (arrow) indicating sclerosis in keeping with impaction syndrome.

positive ulnar variance and an old styloid fracture with evidence of previous surgery (Figure 4a). Moderate oedema was noted in the distal styloid, the proximal ulnar pole of the lunate as well as the styloid fracture fragment (Figure 4b). A diagnosis of ulnar impaction syndrome was made.

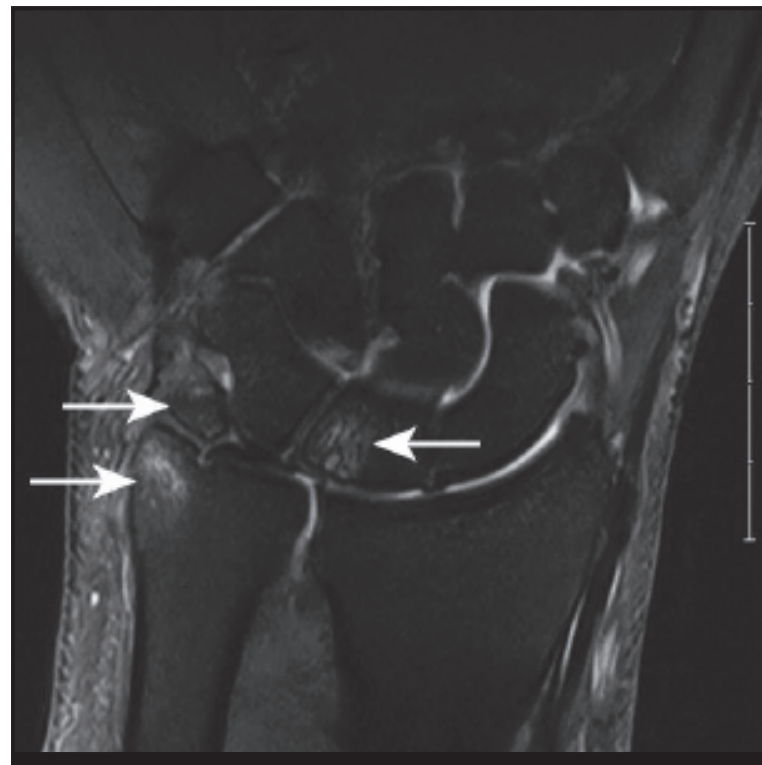

Figure $4 b$. Coronal proton density fat saturated image displaying bone oedema in the distal ulnar, the ulnar styloid fracture fragment as well as the proximal ulnar pole of the lunate (arrows) 


\section{Discussion}

The wrist joint has a unique and complex structure comprising bony structures of the distal radius and ulna, covered with cartilage at the articular surfaces, and the interpositioned ligaments for stability. The relevant joints involved are the distal radio-ulnar, radiocarpal, ulnocarpal and intercarpal joints. All components need to be in a specific relationship to the other structures for normal functioning. One of these important relationships is that between the distal radius and ulna. The relative length of the ulna in relation to the radius at the distal radio-ulnar articular surface is termed ulnar variance - also known as the radio-ulnar index.

Imaging for ulnar variance is done on radiographs with the forearm in the neutral rotation. This is best achieved by placing the upper arm on the X-ray table with the elbow at $90^{\circ}$. The palm should face the cassette. Neutral ulnar variance refers to an equal level of the distal ulna and radius at the articulation site with lunate. The radial styloid process would extend more inferiorly beyond this level by $9-12 \mathrm{~mm}$. In the case where the ulna extends more distally beyond the radius, it is termed positive ulna variance (ulna plus variance) or when projecting more proximally, negative ulna variance (ulna minus variance). ${ }^{2}$

In addition to the relationship of the bony elements, the soft tissue structures are equally important and responsible for normal function. Most notable is the triangular fibrocartilage complex (TFCC). This structure is located between the distal ulna and the proximal carpal row with a medial attachment on the fovea of the ulna and a lateral attachment to the lunate fossa of the radius. The components of the TFCC are the triangular fibrocartilage, radio-ulnar ligament, ulnocarpal ligament, extensor carpi ulnaris tendon sheath and meniscal homologue. It acts as a cushion for the axial forces between the ulna and carpal bones and lends stability to the distal radio-ulnar joint. ${ }^{2}$

The axial load from the forearm is transferred through the wrist to the carpal bones from which approximately $20 \%$ is transferred through the ulnar side. ${ }^{3}$ By increasing the ulna length by $25 \mathrm{~mm}$, the axial load is increased from $20 \%$ to $41.9 \%$, and decreasing the ulna length by $25 \mathrm{~mm}$ decreases the load to $4.8 \% .{ }^{4,5} \mathrm{An}$ increased load transmission across the ulnar side of the wrist can predispose to degenerative conditions including ulnocarpal impaction syndrome. ${ }^{3}$ Ulna variance therefore plays a major role in the mechanical stress transmitted through the wrist.

Chronic degenerative ulnar-sided wrist pathology may be due to overuse with abnormal stress on the ulnar side of the wrist, abnormal joint configuration or a combination of both. The sequelae are an impaction syndrome of the wrist.

There are five impaction syndromes producing ulnarsided wrist pain: ${ }^{1}$

1. Ulnar impaction syndrome (ulnar abutment syndrome/ ulnocarpal loading)

2. Ulnar impingement syndrome

3. Ulnocarpal impaction syndrome secondary to nonunion of the ulnar styloid
4. Ulnar styloid impaction syndrome

5. Hamatolunate impingement syndrome

The above impaction syndromes are all degenerative conditions with differences in pathological processes. Ulnar impaction syndrome is caused by excessive load bearing across the ulnar side of the wrist ${ }^{3}$ presenting typically with symptoms of ulnar-sided wrist pain, decreased range of motion and joint swelling. The pain is mechanical in nature - exacerbated by exercise and relieved by rest. Some activities, e.g. firm gripping, pronation and ulnar deviation of the wrist, cause exacerbation of pain due to the relative increase in ulnar variance. ${ }^{1,6-10}$ The disease process, however, develops over time and patients may have radiological features while being asymptomatic. ${ }^{1}$

A sequence of pathological features of ulnar impaction syndrome occurs depending on the degree and duration of chronic impaction between the ulnar head, the interpositioned TFCC and the ulnar carpus. The pathological radiological features include: fraying (patient described in case 1) or tearing (patient described in case 3 ) of the TFCC, chondromalacia of the ulnar head, lunate or triquetral bone, instability or tear of the lunotriquetral ligament, and a late and advanced feature is osteoarthritis of the distal radio-ulnar joint and ulnocarpal joint. ${ }^{1,6,11,12,13}$

Ulnar impaction syndrome is associated with positive ulna variance but may occur with neutral or negative variance. ${ }^{1,6,8,14}$ Only one patient described in our series had a mildly positive ulnar variance. All other patients had a neutral variance. Other conditions creating positive ulnar variance include previous distal radius fracture or surgery with foreshortening or premature distal radius physeal arrest. ${ }^{1,6,8}$ Pronated grip radiography was described by Tomaino to demonstrate increased variance and the subsequent impaction..$^{8,14}$

The Palmer classification describes the mechanism and degree of injury in the event of TFCC injuries. A distinction was made between class I (traumatic) and class II (degenerative) injuries. Class I traumatic lesions usually cause a sharp disruption of the TFCC. ${ }^{4}$ Class II lesions focus on degenerative ulnar impaction syndrome - specifically on the horizontal part of the TFCC, ulnar head, ulnar carpus and lunotriquetral ligament. ${ }^{3,15}$

Various imaging modalities are available for evaluating ulnar wrist pathology, each with its own advantages and limitations. The initial imaging usually entails radiography anterior-posterior views, lateral views and oblique views with or without pronation and supination and occasionally with carpal tunnel views. ${ }^{8}$

Possible associated X-ray findings of ulnar impaction syndrome include positive ulnar variance, distal radius changes including previous distal radius fracture with malunion, radial head resection or early physeal closure. Other secondary degenerative changes can include subchondral sclerosis (described in all patients in our case series) and also cystic changes of the distal ulna and ulnar poles of the triquetrum and lunate (as described in patient in the patient in case 2). ${ }^{1,6,11,13}$ 
These radiological changes may be subtle in both the early and late stages of the condition. In such cases, when there is a high clinical index of suspicion of ulnar impaction syndrome, further imaging including MRI should be considered for detection of occult disease. ${ }^{16,12}$

Depending on the suspected pathology, other modalities including ultrasound, CT, 99Technetium bone scans, MRI, MR arthrography or conventional arthrography may be used.

In cases of suspected ulnar impaction syndrome, to evaluate for a TFCC injury, the examiner is in fact limited to CT and MRI with or without arthrography or conventional arthrography. Ultrasound, which is known to provide valuable information regarding the soft tissue of the wrist, can detect the TFCC, but the internal structure of the TFCC cannot be accurately demonstrated for final diagnosis and the technique is highly operator-dependent. ${ }^{8}$

The procedure of choice, until recently, to evaluate the TFCC has been arthrography. ${ }^{2}$ It was considered to be the 'gold standard' to diagnose TFCC perforations with tripleinjection arthrography. A relatively high rate of falsenegative findings have however been reported by several authors related to arthrography of the wrist. ${ }^{8}$

The current general belief is that MRI approaches arthrography in accuracy for diagnosing pathology of the TFCC. ${ }^{2}$ MRI has largely supplanted arthrography during the past several years ${ }^{8}$ and the reference standard method for detection of TFCC lesions is now considered to be direct MR arthrography. ${ }^{3}$

Vast improvements of the magnetic quality and surface coils have been made over the last years especially with advances such as the $3 \mathrm{~T}$ magnetic field to achieve higher resolution images. ${ }^{8}$ High resolution is essential for the evaluation of normal and abnormal features of the wrist. ${ }^{8}$

Progressive features of ulnar impaction syndrome can be seen on MRI indicating the degree and extent of the disease process. MR imaging and MR arthrography may be necessary to demonstrate the integrity of the TFCC and lunotriquetral ligaments in patients with radiographic evidence of ulnar impaction syndrome. ${ }^{1,6,12,13}$ The joint cartilage of the ulnar head and ulnar carpus is characterised by fibrillation in the early stages of ulnar impaction syndrome. Further disease progression leads to bone hyperaemia and later to sclerosis. MRI distinguishes this progression with low signal intensity on T1-weighted images and high signal on T2-weighted images demonstrating bone hyperaemia (bone oedema). When sclerosis sets in, T1- and T2-weighted images display low signal intensity. ${ }^{1}$ The normal TFC complex can be seen on coronal T1-weighted images as a low signal intensity biconcave band. TFC complex tears can be seen on T2-weighted images with regions of fragmentation or irregularity of the cartilage with high signal in the areas of interruption. ${ }^{2}$

Direct MR arthrography is useful for assessing the TFCC. Limitations of this technique are the technical difficulty, time, invasiveness and high cost. ${ }^{8}$ On non-gadolinium sequences, the presence of fluid in the distal radio-ulnar joint suggests synovitis and on post-gadolinium sequences, the presence of contrast material in the distal radio-ulnar joint (DRUJ) usually indicates a TFCC tear. ${ }^{8}$

A study by Zanetti et al., however, demonstrated leakage of contrast into the DRUJ of $46 \%$ of asymptomatic wrists of patients undergoing bilateral arthrograms of the wrist when presenting with unilateral wrist pain. ${ }^{16}$ This serves to emphasise that the diagnosis should never be based on an isolated finding but a combination of various radiological signs (bone oedema, sclerosis) and clinical assessment.

With indirect MR arthrography, Schweitzer et al. suggested excellent imaging accuracy for the diagnosis of TFCC tears. ${ }^{17}$ However, Haims et al. showed that indirect MR did not significantly improve the ability to evaluate the central TFCC disc when compared to non-enhanced MRI ${ }^{18}$ As a result, its usefulness for the evaluation of TFCC lesions is controversial. ${ }^{8}$ All our cases were assessed by routine MRI without arthrogram or intravenous gadolinium. Only the patient described in case 3 had an MRI with arthrogram.

Another modality for diagnosis is multidetector computed tomography (MDCT) which is playing an increasingly important role in osseous wrist pathology. ${ }^{8}$ Modern MDCT has significantly higher spatial resolution than MRI. For the detection of TFC and intrinsic ligament lesions, MDCT arthrography yielded comparable results compared to direct MR arthrography. ${ }^{19}$ MDCT provides superior osseous detail, especially with fracture evaluation. ${ }^{8}$ When combined with arthrography, MDCT can provide clear images of the ligamentous structures with detailed bony anatomy of the wrist. ${ }^{8} \mathrm{MDCT}$ is also more exact in the diagnosis and localisation of tears and perforations of the TFCC when compared to conventional arthrography. ${ }^{8}$ The capability of MDCT arthrography in evaluation of soft tissue abnormalities is however limited and often needs to be combined with other modalities such as ultrasound and MRI. ${ }^{8}$

Moser et al. demonstrated in a recent report that for the detection of TFCC tears, lunotriquetral tears and cartilage abnormalities, MDCT arthrography was more accurate than MRI and MR arthrography. ${ }^{20}$ When MR arthrography is contraindicated or MRI is unavailable, MDCT should be considered, as it is a powerful diagnostic tool to diagnose wrist pathology.

\section{Conclusion}

Patients with ulnar-sided wrist pain may often present a diagnostic dilemma for clinicians The symptoms may be intermittent and rather minimal, even in the late stages of the disease. X-ray findings can also vary from prominent to a few non-specific findings. It is, however, very important to maintain a high index of suspicion of the condition as it is a progressive condition which, if left untreated or partially treated by means of a wrong diagnosis, can result in significant morbidity to the patient. Advanced imaging modalities such as MRI with arthrography and MDCT with arthrography are excellent in assisting clinicians with making an accurate diagnosis, as was proven in our four patients presented here. 


\section{Compliance with Ethics Guidelines}

C Liebenberg, MD Velleman and FE Suleman declare no conflict of interest in the writing of this article.

\section{References}

1. Cerezal L, del Pinal F, Abascal F, Garcia-Valtuille R, Pereda T. Imaging findings in ulnar-sided wrist impaction syndromes. RadioGraphics 2002;22:105-21.

2. Greenspan A. Orthopaedic imaging: a practical approach. 4th Edition. Philadelphia: Lippincott Williams and Wilkins, 2004. Chapter 7, Upper limb III: Distal forearm, wrist and hand; p.165-214.

3. Crema M, Marra M, Guermazi A, Roemer F, Bohndorf K. MDCT arthrography features of ulnocarpal impaction syndrome. AJR 2009;193:1376-81.

4. Nakamura R. Diagnosis of ulnar wrist pain. Nagoya J. Med. Sci 2001;64:81-91.

5. Palmer AK, Werner FW. Biomechanics of the distal radioulnar joint. Clin. Orthop. 1984;187:26-35.

6. Friedman SL, Palmer AK. The ulnar impaction syndrome. Hand Clin 1991;7:295-310.

7. Palmer AK, Glisson RR, Werner FW. Ulnar variance determination. J Hand Surg[Am] 1982;7:376-79.

8. Watanabe A, Souza F, Vezeridis P, Blazar P, Yoshioka H. Ulnar-sided wrist pain II. Clinical imaging and treatment. Skeletal Radiol 2010;39:837-857.

9. Mikic ZD. Arthrography of the wrist joint. An experimental study. J Bone Joint Surg Am 1984;66:371-8.

10. Hugo PC, Newberg AH, Newman JS, Wetzner SM. Complications of arthrography. Semin Musculoskelet Radiol 1998:2:345-48.

11. Escobedo EM, Bergman AG, Hunter JC. MR imaging of ulnar impaction. Skeletal Radiol 1995;24:85-90.
12. Imaeda $T$, Nakamura $R$, Shionoya $K$, Makino N. Ulnar impaction syndrome: MR imaging findings. Radiology 1996;201:495-500.

13. Hodge JC, Yin Y, Gilula LA. Miscellaneous conditions of the wrist. In: Gilula LA, Yin Y, eds. Imaging of the wrist and hand. Philadelphia, Pa: Saunders, 1996;523-46.

14. Tomaino MM. Ulnar impaction syndrome in the ulnar negative and neutral wrist: diagnosis and pathoanatomy. $J$ Hand Surg [Br] 1998;23:754-57.

15. Palmer AK. Triangular fibrocartilage complex lesions: a classification. J Hand Surg Am 1989;14:594-606.

16. Zanetti M, Linkous MD, Gilula LA, Hodler J. Characteristics of triangular fibrocartilage defects in symptomatic and contralateral asymptomatic wrists. Radiology 2000;216:840-45.

17. Schweitzer ME, Natale P, Winalski CS, Culp R. Indirect wrist arthography: the effects of passive motion versus active exercise. Skeletal Radiol. 2000;29(1):10-14.

18. Haims AH, Schweitzer ME, Morrison WB, Deely D, Lange $\mathrm{RC}$, Osterman $\mathrm{AL}$, et al. Internal derangement of the wrist: indirect arthrography versus unenhanced MRI imaging. Radiology 2003;227(3):701-707.

19. Omlor G, Jung M, Grieser T, Ludwig K. Depiction of triangular fibrocartilage in patient with ulnar-sided wrist pain: comparison of direct multi-slice CT arthrography and direct MR arthrography. Eur Radiol 2009;19:147-51.

20. Moser T, Dosch JC, Moussaoui A, Dietemann JL. Wrist ligament tears: evaluation of MRI and combined MDCT and MR arthrography. AHR 2007;188:1278-86.

This article is also available online on the SAOA website (www.saoa.org.za) and the SciELO website (www.scielo.org.za). Follow the directions on the Contents page of this journal to access it. 